\title{
RASSEGNA DI STUDI SU IPPOLITO NIEVO
}

\section{STEFANIA SEgatori}

\begin{abstract}
Riassunto: Il saggio presenta un panorama delle edizioni dei testi di Ippolito Nievo e ripercorre la storia della critica nieviana dalle origini all'epoca presente. Al fine di fornire un quadro aggiornato sugli studi nieviani, la rassegna illustra con particolare attenzione l'Edizione nazionale delle opere di Ippolito Nievo, avviata dalla casa editrice Marsilio di Venezia nel 2004. In conclusione, viene considerato il problema della diffusione degli scritti di Nievo con un appunto finale sulle recenti traduzioni delle Confessioni.
\end{abstract}

Invitare le nuove generazioni alla lettura di un "classico" moderno, quale è Ippolito Nievo, e ribadire la sua duplice e decisiva importanza nel processo di formazione dell'identità italiana, è oggi un dovere, dato che il "Giubileo d'Italia", del quale si sente parlare da qualche tempo e che costituisce il cuore delle celebrazioni dei centocinquant'anni dell'Unità d'Italia, è pure l'anniversario della morte dello scrittore friulano, il quale, arruolatosi nei Mille per contribuire anche fisicamente alla causa nazionale, morì proprio nel 1861 per volere di un fato beffardo che gli tolse la vita pochi giorni prima della dichiarazione dell'unificazione della penisola ${ }^{1}$.

Al momento della morte il colonnello Ippolito Nievo, Cacciatore delle Alpi e Vice-intendente dell'Esercito meridionale, era ai più un perfetto sconosciuto $^{2}$ e restò tale anche negli anni successivi, sia per la censura e il sospetto venutosi a creare intorno al suo romanzo maggiore, Le Confessioni

${ }^{1}$ Sulla morte del poeta soldato: Nievo, Il prato in fondo al mare, Zinna, Come un sogno incredibile. Ipotesi sul caso Nievo; Samaritani, Ippolito Nievo. I giorni sommersi; Cammilleri, Sherlock Holmes e il misterioso caso di Ippolito Nievo; Zinna, Il Caso Nievo. Morte di un Garibaldino; Glori, La tragica morte di Ippolito Nievo. Il naufragio doloso del piroscafo "Ercole". Coinvolgimento e responsabilità della Massoneria nella caduta del Regno delle Due Sicilie, Ruffilli, Lisola e il sogno. Dati inediti, come le condizioni metereologiche del 4 marzo 1861, sono stati reperiti dalla Samaritani e sono disponibili sul sito web all'indirizzo http://www.ippolitonievo.info/MorteNievo.htm. Rinvio, infine, ad una recente puntata del programma La storia siamo noi andata in onda su Rai3 il 4 marzo 2011: Ippolito Nievo inchiesta sui Mille di Andrea Bignami (http://www.rai.tv).

2 I primi interessanti riferimenti al Nievo si hanno in Molmenti, Impressioni letterarie. 
d'un Italiano', sia per l'analisi negativa formulata da Benedetto Croce (racconto "difettoso" nel disegno e incompiuto nella "forma artistica" 4 ). Una prima parentesi si rende subito necessaria. Secondo Calvino (si vedrà in seguito come un dialogo a distanza unisca quest'ultimo al Nievo), la lettura delle Confessioni doveva essere "pericolosa" per i lettori del tempo, perché l'autore metteva spesso in relazione la parola "patria" ai concetti di pace, fraternità, giustizia, solidarietà, consapevolezza intellettuale, studio. Riflessione che la dice lunga sulla prima edizione del capolavoro nieviano, pubblicato postumo nel 1867 con il titolo Le Confessioni d'un ottuagenario ${ }^{5}$. Cinque anni dopo, la scrittrice veneta Luigia Codemo metteva in guardia gli italiani dalla lettura di questo romanzo: una letteratura di quel genere, sosteneva, "non ha ideale", "è senza avvenire"6. Due, quindi, i livelli di censura: il titolo e la protagonista femminile. Ricordo che ad insistere per la pubblicazione presso Felice Le Monnier, dopo tanti rifiuti a Milano, fu Erminia Fuà Fusinato, moglie di Arnaldo e poetessa di discreto successo all'epoca, la quale aveva ricevuto il manoscritto da Bice Gobio Melzi, poco prima di morire nel 1865 . Il pregiudizio garibaldino che andava diffondendosi tra i ceti moderati e l'affievolirsi dell'ideologia nazionalistica smorzarono l'entusiasmo per la memorialistica risorgimentale, legittimando così le esitazioni dell'editore fiorentino. D'altronde, lo stesso Nievo temeva una censura proprio sul titolo. La testimonianza storico-politica di Carlino viene prudentemente camuffata dalla ricordanza sentimentale di un "ottuagenario". Per quanto riguarda la Pisana, condannata dalla Società italiana contro le cattive letture (presidente onorario Gino Capponi, effettivo Niccolò Tommaseo), che schedava il romanzo nieviano fra i testi "sconsigliati alle famiglie e alle biblioteche popolari" (CI, IX), secondo la

3 Le citazioni sono tratte da Nievo Ippolito. Le Confessioni d'un Italiano, a cura di M. Gorra. Milano: Arnoldo Mondadori, 2006 e, d'ora in poi, saranno date da questa edizione direttamente nel testo o in nota con l'abbreviazione $C I$ seguita dal numero della pagina.

4 Croce, "Ippolito Nievo" in La letteratura della nuova Italia, 131-35. Il critico ammirava Nievo come uomo che aveva profuso il meglio di sé nella vita attiva. Lartista ne usciva ridimensionato e, paradossalmente, ancora prigioniero del circolo biografia-poesia innescato dal Mantovani: "Croce era responsabile, oltre che di alcuni giudizi come quello sulla 'semplicità fredda' dell'uomo Nievo, della perentoria riduzione della complessità delle Confessioni ad alcuni temi e motivi” (Colummi Camerino, Introduzione a Nievo, 124).

5 Calvino, I libri degli altri. Lettere 1947-1981.

6 Cfr. Codemo Gerstenbrandt, Fronde e fiori del Veneto letterario in questo secolo, 142-145. 
Codemo "l'affetto per una tal femmina è così da non mostrarsi con troppa evidenza al pubblico". Non c'è da stupirsi che i benpensanti fossero sconvolti dalla più giovane delle contessine di Fratta ${ }^{7}$. La Pisana rappresentava un'anomalia rispetto alle altre eroine ottocentesche, ma il "ritegno" serbato dalla critica dell'epoca non dipendeva soltanto dalla sua conturbante presenza, intollerabile per i moralisti, ma, piuttosto, da alcune anticipazioni significative che avrebbero caratterizzato il pensiero politico dell'ultimo Nievo: l'interesse per le condizioni economico-sociali delle classi popolari; la sicurezza del lavoro; la libertà e l'uguaglianza; la fine del potere dell'aristocrazia e dell'aristocrazia del denaro.

Tornando alla storia della critica nieviana, secondo la Colummi Camerino, Nievo alla sua morte era conosciuto solo in ambienti specialistici: "la sua piccola notorietà è legata alla produzione dei Versi friulani e alla recensione che Tenca ne ha fatto sul Crepuscolo. La sua immagine, come emerge negli articoli milanesi, è quella di un giovane di serio impegno che, messosi sulla scia di Giusti, ricerca una sua autonomia nell'arduo mélange di toni seri e giocosi, nella difficoltosa resa formale di 'un'ispirazione ricca capricciosa saltellante'. Del narratore, invece, nessuna notizia”.

La prima biografia nieviana fu firmata da Dino Mantovani nel 1900, seguita da quella di Giuseppe Solitro nel 1936. Ma la natura autobiografica delle Confessioni (non poteva essere altrimenti) iniziò pian piano a suggerire nuove analisi critiche9. Agli inizi del Novecento, numerosi intellettuali si avvicinarono all'opera nieviana, cercando di collocare e rivalutare lo

7 Nel romanzo è la protagonista femminile a trionfare su tutti: volubile e inafferrabile, dapprima bambina terribile, poi giovane generosa e capricciosa, infine, donna che compie miracoli d'abnegazione; sicuramente, uno dei personaggi più psicologicamente ricchi della letteratura italiana. La Pisana è donna fatta sin dall'infanzia. Carlino la descrive come "una farfalla che non può ristar due minuti sulla corolla d'un fiore" ( $C I, 115)$. L'identità di questa nuova tipologia di eroina letteraria è esplicita fin dalla prima riga del romanzo: perfetta compresenza dei contrari che la compongono.

8 Colummi Camerino, Introduzione a Nievo, 123.

9 L'operazione che Romagnoli fece per il volume di Opere della collana "La letteratura italiana. Storia e testi” di Ricciardi si fondava su alcuni criteri divenuti irrinunciabili nell'interpretazione del lavoro nieviano. Di qui i suggerimenti, anche linguistici, per una rilettura dell'opera di Nievo che, invece di attribuire a "sciatteria" le numerose forme dialettali, le addebitava alla "programmatica velocità d'espressione" di un autore che si affidava al suo "naturale linguaggio" di lombardo-veneto. Romagnoli avviava così anche un recupero dell'opera integrale di Nievo nel rispetto dei suoi valori testuali e biografici, psicologici e storici. 
scrittore all'interno di correnti e generi ben definiti. Non vanno al di là della curiosità letteraria il progetto pirandelliano, risalente presumibilmente al 1911 e rimasto incompiuto, di una sceneggiatura cinematografica delle Confessioni di cui ci informa Lucio D'Ambra nell'articolo Sette anni di cinema, apparso sulla rivista Cinema il 25 gennaio 1937; o la conferenza su Nievo tenuta dal giovane Moravia nella primavera del 1936 alla Columbia University ${ }^{10}$. Testimoni tutt'al più, nel primo caso, di attenzione verso l'umorismo insito nelle Confessioni ${ }^{11}$, di cui Pirandello aveva già accennato nel noto saggio del 1908 e di come esso sia sfuggito alla critica; nel secondo caso, di una volontà narrativa di genere ricostruttivo a favore di una prosa fatta di interessi sociali, didattici, storici e psicologici. Inevitabile l'entusiasmo per il combattente Ippolito Nievo della stampa fascista, a partire dall'anno del centenario della nascita (1931) e per tutta la durata del Regime. Appartiene a questo periodo il profilo biografico di Alfredo Fabietti nella collana "La centura di ferro. La pattuglia dei grandi spiriti, n. 35" (1937).

Il secondo dopoguerra, con la nozione di cultura engagée e l'attitudine al realismo narrativo, ha registrato un intensificarsi degli studi nieviani con una particolare attenzione agli scritti politici e alla produzione campagnola. Anche se la figura di Nievo non raccoglieva consensi tra i protagonisti

$10 \mathrm{Ne}$ dà notizia l'autobiografia in forma di intervista rilasciata ad Alain Elkann. Per allontanarsi da un Paese che incominciava a rendergli la vita difficile, Moravia partì per New York nel 1935, invitato da Giuseppe Prezzolini, allora direttore della Casa Italiana della Columbia University di New York; qui tenne una conferenza sul romanzo italiano, discutendo su Nievo, Manzoni, Verga, Fogazzaro e D’Annunzio. A New York Moravia rimase dall'ottobre 1935 fino al maggio del 1936 (con una breve parentesi messicana per sfuggire al freddo intenso invernale che si era abbattuto sugli Stati Uniti). Scrive Moravia: "Diremo dunque: D’Annunzio non è romanziere perché le sue pagine di romanzo fanno bella figura in tutte le antologie; Nievo invece è romanziere perché difficilmente brani del suo libro inseriti in un'antologia possono dare un'idea compiuta e valorosa della sua arte. Avverto a scanso di equivoci che qui non faccio questione di valore artistico sebbene di organicità" (Moravia, "Il romanzo è morto, viva il romanzo". L'articolo non è mai stato antologizzato).

11 Leggendo attentamente le Confessioni si percepisce un umorismo di fondo, tipico della tradizione settecentesca. Basta accennare alla diligenza con cui gli scrittori del secolo precedente ritraevano i loro personaggi con una perizia calligrafica che riproduceva in modo verosimile tutta la geografia di un universo. Ma anche per quella febbrile smania moralistica di indagare le anime altrui per riproporle isomorfe nero su bianco. Nella ponderata consapevolezza risiede la genialità delle Confessioni. 
del Neorealismo, poco inclini a trattare la sua produzione, è altrettanto vero che ad imporsi non fu l'immagine del narratore impegnato e realista, quanto il suo profilo di scrittore d'infanzia e di paesaggi che segna l'esordio di due scrittori di assoluto rilievo nella letteratura del secondo dopoguerra: Pier Paolo Pasolini e Italo Calvino. Quest'ultimo confessò pubblicamente la sua passione per lo scrittore friulano ${ }^{12}$ :

[Nievo] conosceva cos'era avventura, e storia familiare, e grandezza e decadenza sociale, e vita umana e presenza della donna nella vita dell'uomo, e paesaggio natale, e trasfigurazione della memoria in continua presenza reale: il generoso, il giovane, il fluviale $\mathrm{Nievo}^{13}$.

Io ho sempre avuto coscienza di prendere dei prestiti, di fare degli omaggi, e in questo caso fare omaggio a un autore significa appropriarsi di qualcosa che è suo. Fin dalle prime cose che ho scritto mi proponevo dei punti d'incontro tra due modelli molto diversi, per esempio Pinocchio e Faulkner, Hemingway e Ippolito Nievo ${ }^{14}$.

Non dimentichiamoci che lo slancio del Risorgimento italiano ha avuto in letteratura una sola eco veramente poetica: e sono le giornate avventurose del Carlino di Nievo tra gli spalti e i fossi attorno al decrepito Castello di Fratta ${ }^{15}$.

Amo Nievo perché l'ho riletto tante volte divertendomi come la prima ${ }^{16}$.

Il Castello di Fratta è il vestigio d'una società decrepita, ma è anche il luogo dell'infanzia: perciò è il primo che un romanzo ci rappresenti con calore e con umore, con affetto ${ }^{17}$.

Quali autori ebbero maggior peso nella tua formazione di scrittore? [...] Dirò subito: Le Confessioni d'un ottuagenario di Ippolito Nievo, l'unico romanzo italiano dell'Ottocento dotato d'un fascino romanzesco paragonabile a quello che si trova con tanta abbondanza nelle letterature straniere. Un episodio del mio primo romanzo Il sentiero dei nidi di ragno s'ispira all'incontro di Carlino e di Spaccafumo. Una vaga atmosfera da Castello di Fratta è evocata nel Visconte dimezzato. E Il barone rampante ricalca il romanzo di Nievo nell'arco d'una vita che copre lo stesso perio-

12 Nozzoli, Immagini di Nievo nel Novecento.

13 Calvino, Saggi 1945-1985, 1508.

14 Calvino, Saggi 1945-1985, 1805.

15 Calvino, Saggi 1945-1985, 41.

16 Calvino, Saggi 1945-1985, 1529.

17 Calvino, Saggi 1945-1985, 1646. 
do storico tra Sette e Ottocento e gli stessi ambienti sociali; per di più, il personaggio femminile ha per modello la Pisana ${ }^{18}$.

È la ricerca di tipo ariostesco ${ }^{19}$, come intuì giustamente Pavese, l'elemento di partenza che riconduce Calvino a $\mathrm{Nievo}^{20}$, dato che per entrambi l'Ariosto era l'immagine della modernità e l'Orlando un esempio di meraviglioso, l'elemento fantastico percepito come normale. La fedeltà nieviana di Calvino è testimoniata da numerose lettere ed interviste, ma la ricerca sulla vicenda dell' attraversamento di Nievo da parte di Calvino è ancora in fieri ${ }^{21}$. Un nostalgico intrecciarsi di riferimenti lega, infatti, Il barone rampante alle Confessioni22.

18 Calvino, Saggi 1945-1985, 2920.

19 Sull'Ariosto Calvino pubblicò L“'Orlando Furioso" di Ludovico Ariosto raccontato da Italo Calvino e "La struttura dell'Orlando" in Perché leggere i classici, 68-77. Qualcosa di affine aveva scritto Sanguineti, "La macchina narrativa dell'Ariosto" in Il chierico organico. Scritture e intellettuali, 65-71.

20 Dichiara Pavese a proposito de I sentieri dei nidi di ragno: "C’è qui dentro un sapore ariostesco. Ma l'Ariosto dei nostri tempi si chiama Stevenson, Kipling, Dickens, Nievo, e si traveste volentieri da ragazzo" (L'Unità, 26 ottobre 1947).

21 Carlino prima, Cosimo poi, diventano portatori di un' esemplarità imperfetta, se non altro nella loro capacità di riconoscere schiettamente le proprie virtù e le proprie miserie, in quel loro saper vivere contemporaneamente il mito della gioventù e la pazienza di una disincantata maturità, senza venir meno ad una serena moralità. Il Furioso, primo romanzo moderno, proprio perché percorre un mondo visto e descritto con un'ottica moderna, la cui vasta totalità è un problema da afferrare e ricomporre (non un'armonia perfetta, ma un'eterotopia), è il punto di partenza per comprendere il modo di guardare dei due protagonisti, costantemente alla ricerca di una forma-paesaggio che dia il contorno di un'epoca, per trovare corrispondenze nello spazio usuale e per ricercare se stessi o nel piacere di nuove scoperte o nel proprio disorientamento. Il poema ariostesco è romanzo nella prospettiva di uno spaesamento o di uno straniamento. L'aventure ha lo scopo di elevare il destino riconciliando individuo e comunità. Perdersi per poi riconoscersi in un disegno comune: questo fanno i personaggi dell'Ariosto agli occhi di Nievo e Calvino (si pensi alla vicenda di Astolfo sulla Luna).

22 Larco della vita di Cosimo di Rondò copre pressappoco gli stessi anni di quella di Carlino di Fratta; non manca la galleria degli eccentrici nobilotti di provincia, tra cui un familiare vestito alla turca (come in Nievo il redivivo padre di Carlino); Viola può essere considerata una sorella minore della Pisana, vera libertina ancien Régime; e gli echi della Rivoluzione, gli Alberi della Libertà, perfino l'incontro con Napoleone in persona sono elementi comuni ai due testi. Ma il ricordo dell'appassionata visione del mondo di Nievo non fa altro che risaltare la stilizzazione grottesca ed ironica del Barone rampante. 
La critica nieviana conobbe un forte sviluppo nel periodo post-bellico, probabilmente anche per l'avvicinarsi del centenario della morte ${ }^{23}$. Dopo il volume di Romagnoli si sviluppò la cosiddetta critica sociologica: Ferruccio Ulivi, Carlo Bascetta, Vincenzo Gentili. Il contributo più interessante, ad oggi indispensabile per chiunque voglia approfondire il genere rusticale in Nievo, è quello einaudiano del 1956. La resa del testo integrale del Novelliere campagnuolo, ad opera di Iginio De Luca, che nell'introduzione al volume ricostruiva le coordinate italiane ed europee della produzione rusticale e il dibattito sociale e letterario che l'aveva accompagnata negli anni Quaranta e Cinquanta, ha avuto il merito di restituire organicità ad un testo letto, fino ad allora, quasi esclusivamente attraverso Il Varmo, quintessenza e prefigurazione dell'idillio maggiore delle Confessioni.

Nel 1962 Emilio Faccioli curò un'edizione completa del teatro nieviano, che conteneva gli inediti Emanuele, Gli ultimi anni di Galileo Galilei, Pindaro Pulcinella, I beffeggiatori. In appendice, due abbozzi per teatro: Don Giovanni e Saturno redituro. Il volume fu stampato in un numero esiguo di esemplari, ritirati subito dal commercio dall'autore stesso. Solo una decina di copie sono giunte ai nostri giorni ed alcune biblioteche italiane ne conservano una fotocopia ${ }^{24}$.

Successivamente, l'attenzione della critica si è concentrata sull'impegno sociale del Nievo. Il culmine di questo processo di lettura sociologicamente orientata, dopo le esperienze di Gianni Scalia e Franco Mollia, è rappresentato dal volume di Arnaldo Di Benedetto, Nievo e la letteratura campagnola, inserito in una storia letteraria, che toglie di fatto il primato alle Confessioni e fa del Nievo il massimo esponente italiano della letteratura rusticale ${ }^{25}$.

Al fine di una ricostruzione quanto più possibile completa della criti-

23 Numerosi i volumi, saggi ed articoli apparsi nel 1961, in occasione del centenario della morte. Tra gli altri, si ricordano: Nievo, Quaderno di traduzioni; Nievo, Novelle romantiche; Nievo, Il Varmo; Nievo, Le lucciole; Nievo, Lettere garibaldine; Titta Rosa, Nievo. Uomo e poeta del Risorgimento; Mostra dei cimeli di Ippolito Nievo nel centenario 1861-1961.

24 Nievo, Teatro. Va detto che nei primi del Novecento uscirono per la casa editrice Carabba le tragedie Spartaco e I Capuani, entrambe a cura di Vincenzo Errante.

25 Lo studioso fa risalire le origini della narrativa rusticale al lungo racconto Nunziata del milanese Giulio Carcano (1852), che prepara la narrativa storica al passaggio alla narrativa sociale: "In tal modo", per dirla alla Lukács, si passa "dalla rappresentazione della storia passata alla rappresentazione del presente come storia" (Di Benedetto, Nievo e la letteratura campagnola, 3). 
ca nieviana, vanno ricordati i recuperi testuali (Sergio Romagnoli, Ferruccio Ulivi, Emilio Faccioli) così come una critica di fonti particolarmente attenta a mettere a fuoco la formazione dell'autore (Cesare Bozzetti, Armando Balduino) e gli influssi della letteratura italiana e straniera sulla sua opera (Glauco Natoli). I risultati della critica stilistica hanno, invece, catalizzato l'attenzione sugli aspetti formali più significativi, avviando il discorso relativo alla struttura delle Confessioni e alla lingua della narrativa nieviana. Oltre agli studi di Folco Portinari ed Arnaldo di Benedetto, resta insuperata l'egregia analisi linguistica dell'epistolario nieviano a cura di Pier Vincenzo Mengaldo.

Non sviluppata risulta, infine, la riflessione sulle strutture narrative della produzione nieviana da un punto di vista strettamente psicanalitico, campo quasi vergine all'attenzione dei critici. Unico caso degno di nota è il lavoro di Giuliana Sanguinetti Katz ${ }^{26}$ : l'influsso della madre nella vicenda personale dell'autore avrebbe contribuito, secondo la studiosa, a determinare un atteggiamento di degradazione della donna in quanto oggetto d'amore 27 .

Dopo il fallimento di ben due progetti di Edizione Nazionale delle opere di Nievo (Ricciardi ed Einaudi) negli anni cinquanta-sessanta e settanta-ottanta del secolo scorso, la casa editrice veneziana Marsilio ha avviato nel 2004 una nuova Opera Omnia con lo scopo di recuperare ogni singolo testo o scritto nieviano. L'autorevolezza del comitato scientifico ne garantisce già la completa realizzazione (Pier Vincenzo Mengaldo, Armando Balduino, Simone Casini, Cesare De Michelis, Claudio Gallico, Gilberto Pizzamiglio, Piermario Vescovo, l'Accademia nazionale Virgiliana di Mantova). L'Edizione Nazionale si presenta con il formato friendly del tascabile, ma non rinuncia ad un ricco e puntuale corredo: ampie introduzioni, note al testo ed apparati critici curati nei minimi dettagli; in questo modo, le opere conservano tutta la loro individualità, senza essere schiacciate dal giganteggiare delle Confession ${ }^{28}$. La collana sembra non conosce-

26 Sanguinetti Katz, The Uses of the Myth in Ippolito Nievo e "A psychoanalytic interpretation of the life of Ippolito Nievo."

27 Ricordo che la Pisana rappresentava, nelle Confessioni, un'infrazione intollerabile al conformismo moralistico e tradizionalistico arroccato sull'arcaicità del costume, sul presupposto naturale dell'egemonia borghese, sui bastioni confessionali; e un'infrazione anche al conformismo specificatamente letterario.

28 Per la collocazione degli autografi nieviani rinvio alla sezione "Collocazione degli autografi di Ippolito Nievo nelle Biblioteche, nei Musei e presso privati”. In Mostra dei cimeli di Ippolito Nievo nel centenario 1861-1961, 43-49. 
re ritardi: luminosa eccezione nel panorama delle grandi imprese editoriali, l'Edizione nazionale di Nievo, presieduta da Pier Vincenzo Mengaldo, si distingue per l'elevata qualità scientifica e per la rapidità di realizzazione.

I volumi già editi meritano di essere ricordati in questa sede per la penetrante capacità di analisi critica e di osservazione che non trascura mai l'inquadramento storico-politico dei testi, per le notizie bio-bibliografiche e per le puntuali annotazioni di tipo linguistico-etimologico che accompagnano la lettura delle opere riproposte nella loro originalità. Piermario Vescovo nelle Commedie offre al lettore i due testi comici che ci sono giunti in redazione completa: Pindaro Pulcinella si concentra sul contrasto tra gli ideali del povero poeta romantico Valerio e il ruolo che la letteratura riveste in un mondo popolato da burattini; Le invasioni moderne rappresentano uno spaccato burlesco dell'alta società meridionale, dove Nievo riprende gli schemi allusivi della commedia goldoniana ${ }^{29}$. Rileggere i Drammi giovanili, curati da Maurizio Bertolotti, orienta la critica nell'approfondimento della questione prettamente politica in Nievo e del cambiamento della sua opinione circa la linea di condotta da adottare nella lotta. L'Emanuele, dedicato all'amico israelita Emanuele Ottolenghi, mette in scena le prove affrontate all'indomani del Quarantotto da un giovane ebreo molto ricco che aspira a "occupare un posto nella società". Ne Gli ultimi anni di Galileo Galilei, invece, si ripercorrono le vicende salienti del conflitto tra lo scienziato e il Sant'Uffizio. I due drammi presentano un'innegabile unità ideale che chiarisce il percorso politico e il successivo ripensamento del Nievo e mostrano un atteggiamento non conformista del giovane scrittore, aperto ai principi del liberalismo e partecipe attivo dei nuovi movimenti. Per quanto riguarda il Nievo romanziere, si segnala l'uscita dell'Angelo di bontà e del Conte Pecorajo, entrambi riproposti in due versioni (sia secondo l'editio princeps che sulla base dei manoscritti). Nel primo scritto, ambientato nella Venezia del 1749 , Nievo rielabora il genere del romanzo storico, facendo incarnare all'Inquisitor Formiani la decadenza dei nobili veneziani, mentre nel secondo lo scrittore ritrae il mondo contadino nella sua dimensione "politica", senza alcuna retorica bucolica. Si tratta di uno scritto che, dando conto di una questione locale e periferi$\mathrm{ca}$, riverbera un dato nazionale. Con il Conte Pecorajo e i racconti del

${ }^{29}$ La commedia I Beffeggiatori è stata pubblicata, sulla base del manoscritto ritrovato, in soli cento esemplari nel 2006 per la Pontremoli di Milano. In questa commedia in quattro atti sulla crisi dell'aristocrazia palermitana, Nievo perfeziona la sua forza comica, attraverso dialoghi disinvolti e vivamente coloriti, e una tecnica allusiva capace di spingersi fino agli estremi del sibillino (pratica che gli autori risorgimentali andavano da tempo sperimentando). 
Novelliere campagnuolo, infatti, il giovanissimo Nievo entra a pieno titolo nel dibattito europeo sulla poetica campagnola-rusticale, offrendo una risposta convincente alle questioni sollevate da più importanti modelli letterari (Manzoni, Sand, Carcano).

È questa corposa rinascita di studi che permette alla critica contemporanea di proporre nuovi percorsi di lettura sia della vicenda biografica di Nievo che della sua narrativa. Un aspetto mi preme sottolineare: l'attività intellettuale dello scrittore friulano è sì specifica di un'età di transizione, di una visione del mondo sbilanciata tra passato e presente e in bilico tra presente e futuro, ma anche frutto di una commistione di generi, di temi, di forme. Tra radicamento nella tradizione e spinta verso la modernità, vi è, nella narrativa nieviana, una costante duplicità di unità distinte, ma correlate: la campagna e la Nazione, il senso del luogo natio e il viaggio, le piccole mille patrie e il sogno dell'Unità, il passato, rievocato con frequenti abbandoni nostalgici, e il futuro, impresso nello sguardo sicuro della giovinezza, la memoria e la speranza. Per Nievo il problema più assillante non era introdurre elementi di discontinuità nella tradizione pregressa del romanzo, ma al contrario rilanciarne la morfologia, riplasmando in chiave d'attualità il paradigma egemone, quel componimento misto di storia e d'invenzione su cui si era basata la lezione manzoniana. Seguendo questa chiave di lettura, si può comprendere meglio la definizione che Mengaldo ha dato del capolavoro nieviano, ovvero quella di romanzo europeo, nell'accezione specifica di novel, nella cui ampia architettura convergono le suggestioni più disparate, fuori da schemi vetusti o disegni preordinati. Gli scrittori italiani dell'epoca si trovarono, come nel caso nieviano, a rappresentare una realtà politica in cui l'unità nazionale o doveva ancora essere conquistata o era stata appena raggiunta. Da questa specifica condizione derivò uno sperimentalismo ancorato alla tradizione, ma, allo stesso tempo, proiettato verso un rinnovamento di fondo con un chiaro e preciso intento formativo-educativo ${ }^{30}$.

Mi sia concessa un'ultima considerazione sulle recenti traduzioni delle Confessioni, ritenute oggi dagli studiosi il testo simbolo dei 150 anni dell'Unità d'Italia. In area germanofana si segnala la traduzione di Barbara

30 La volontà di creare un romanzo si è dovuta necessariamente scontrare con volontà centrifughe, costantemente presenti nell'ideologia degli autori stessi e fattori condizionanti le manifestazioni della prosa narrativa (il riferimento è alle culture periferiche e sub-nazionali, stratificate, mai interamente chiuse, anzi a volte direttamente aperte verso la cultura straniera, soprattutto nel caso delle letterature di confine). In questa prospettiva, l'opera di sintesi tra localismo e progresso del Nievo può essere considerata una delle risposte più innovative dell'epoca. 
Kleiner Bekenntnisse eines Italieners (Zurigo: Menasse, 2005) ${ }^{31}$. Si tratta di una resa che rispetta la polifonia stilistica dell'originale, liberandolo da quelle espressioni obsolete del tedesco che ne avevano ostacolato la fortuna. Un'ottima traduzione che nel 2007 è stata premiata con il Premio della Fondazione Kunstverein Nordrhein-Westfalen di Düsseldorf, uno dei riconoscimenti tedeschi più prestigiosi per la traduzione. In lingua spagnola, un'importante iniziativa giunge dalla casa editrice Acantilado di Barcellona che nel 2008 ha pubblicato, per la prima volta in Spagna, Las confesiones de un italiano (traduzione di José Ramón Monreal ${ }^{32}$ ). La notizia, in realtà, è un'altra. Babelia, il supplemento culturale del quotidiano El Pais, ha chiesto a cinquantasette critici e giornalisti suoi collaboratori, di indicare $\mathrm{i}$ migliori quaranta libri apparsi nel 2008. A sorpresa, al trentatreesimo posto si trova un testo difficilmente sospettabile di poter entrare in una simile graduatoria: Le Confessioni d'un Italiano di Ippolito Nievo. Ecco perché, sulla base sia delle iniziative della casa editrice Marsilio (i volumi dell'Edizione nazionale, che come si è visto si rivolgono ad un pubblico non solo di specialisti, e la recentissima biografia della Chaarani-Lesourd ${ }^{33}$ ) che delle attuali traduzioni si può realmente sperare in una nuova stagione di ricezione letteraria di Ippolito Nievo, anche fuori dei confini nazionali.

Università “G. D’AnNunzio” Chieti-Pescara

\section{Opere Citate}

Balduino, Armando. "Ippolito Nievo letterato e uomo nelle Lettere". Belfagor, 38 (1983): 559-69.

Bozzetti, Cesare. La formazione del Nievo. Padova: Liviana, 1959.

Calvino, Italo. I libri degli altri. Lettere 1947-1981. A cura di Giovanni Tesio. Torino: Einaudi, 1991.

"La struttura dell'Orlando". In Perché leggere i classici. Milano: Mondadori, 1995. 68-77.

L"Orlando Furioso" di Ludovico Ariosto raccontato da Italo Calvino. Torino: Einaudi, 1970.

31 Si segnalano le due traduzioni in lingua tedesca delle Confessioni uscite precedentemente l'edizione citata nel testo Nievo: Erinnerungen eines achtzigjährigen e Pisana oder die Bekenntnisse eines Achtzigjährigen.

32 Della traduzione ne ha dato notizia El País nell'articolo El gran fresco de la unidad italiana, dove vengono riportati alcuni passi della prefazione di Claudio Magris. Oltre a El País, anche le pagine di $A B C$ e La Vanguardia hanno dedicato articoli al Nievo. Sul piano critico, invece, rinvio ai contributi di due studiosi argentini Beatriz Curia e Alejandro Patat.

33 Chaarani-Lesourd, Ippolito Nievo. Uno scrittore politico. 
—. Saggi 1945-1985, a cura di M. Barenghi. Milano: Mondadori, 1995.

Cammilleri, Rino. Sherlock Holmes e il misterioso caso di Ippolito Nievo. Cinisello Balsamo: San Paolo, 2000.

Chaarani-Lesourd, Elsa. Ippolito Nievo. Uno scrittore politico. Venezia: Marsilio, 2011.

Ciceri, Luigi. "Versi satirici inediti di Ippolito Nievo". Sot la nape, 1 (1961): 41-48.

Codemo Gerstenbrandt, Luigia. Fronde e fiori del Veneto letterario in questo secolo. Venezia: Tipografia Cecchini, 1871.

Colummi Camerino, Marinella. Introduzione a Nievo. Roma-Bari: Laterza, 1991.

Croce, Benedetto. "Ippolito Nievo". In La letteratura della nuova Italia, vol. I. Bari: Laterza, 1914. 131-35.

D’Ambra, Lucio. "Sette anni di cinema”. Cinema (25 gennaio 1937).

Di Benedetto, Arnaldo. Nievo e la letteratura campagnola. Roma-Bari: Laterza, 1990.

Elkann, Alain. Vita di Moravia. Milano: Bompiani, 1990.

Fabietti, Alfredo. Ippolito Nievo. Milano: Oberdan Zucchi, 1937.

Glori, Cesaremaria. La tragica morte di Ippolito Nievo. Il naufragio doloso del piroscafo "Ercole". Coinvolgimento e responsabilità della Massoneria nella caduta del Regno delle Due Sicilie. Chieti: Solfanelli, 2010.

Mantovani, Dino. Il poeta soldato. Ippolito Nievo (1831-1861) da documenti inediti. Milano: Treves, 1900.

Mengaldo, Pier Vincenzo. L'epistolario di Nievo. Analisi linguistica. Bologna: Il Mulino, 1987.

Mollia, Franco. Ippolito Nievo. Firenze: La Nuova Italia, 1968.

Molmenti, Pompeo G. Impressioni letterarie. Milano: Battezzati, 1875.

Moravia, Alberto. "Il romanzo è morto, viva il romanzo". Prospettive, 4.5 (15 maggio 1945): 5.

Mostra dei cimeli di Ippolito Nievo nel centenario 1861-1961. Udine, Sala Aiace, 1631 dicembre 1961. Udine: Doretti, 1961.

Natoli, Glauco. "Réminiscences françaises dans Le Confessioni di un italiano". Revue de Littérature comparée, 25 (1951): 209-31.

Nievo, Ippolito. Angelo di bontà. Storia del secolo passato, a cura di A. Zangrandi. Venezia: Marsilio, 2007. Angelo di bontà. Storia del secolo passato. Edizione critica dall'autografo del 1855, a cura di A. Zangrandi e P. V. Mengaldo. Venezia: Marsilio, 2009.

—. Bekenntnisse eines Italieners, traduzione di B. Kleiner. Zurigo: Menasse, 2005. Commedie, a cura di P. Vescovo. Venezia: Marsilio, 2004.

- Drammi giovanili. Emanuele e Gli ultimi anni di Galileo Galilei, a cura di M. Bertolotti. Venezia: Marsilio, 2006.

—. Erinnerungen eines achtzigjährigen, traduzione di I. Kurz. Leipzig: Grunow, 1877.

—. I Beffeggiatori, a cura di G. Gulli. Milano: Pontremoli, 2006.

—. I Capuani: tragedia, a cura di V. Errante. Lanciano: Carabba, 1914.

-. Il Conte Pecorajo. Storia del nostro secolo. Testo critico secondo i manoscritti del 1855-56, a cura di S. Casini. Venezia: Marsilio, 2010. 
—. Il Varmo, a cura di V. Branca. Firenze: Le Monnier, 1961.

- Las confesiones de un italiano, traduzione di J.R. Monreal. Barcellona: Acantilado, 2008.

—. Le Confessioni d'un Italiano, a cura di M. Gorra. Milano: Arnoldo Mondadori, 2006.

—. Le Lucciole, poesie scelte a cura di D. Selvatico Estense, con uno scritto di R. Bacchelli. Milano: All'insegna del pesce d'oro, 1961.

—. Lettere garibaldine, a cura di A. Ciceri. Torino: Einaudi, 1961. Novelle romantiche, a cura di A. Martello. Milano: A. Martello, 1961.

—. Novelliere campagnuolo, a cura di I. De Luca. Torino: Einaudi, 1956.

—. Opere, a cura di S. Romagnoli. Milano-Napoli: Ricciardi, 1952.

—. Pisana oder die Bekenntnisse eines Achtzigjährigen, traduzione di C. Birnbaum.

Francoforte: Weisbecker, 1956.

—. Quaderno di traduzioni, a cura di I. De Luca. Torino: Einaudi, 1961.

—. Scritti politici e storici, a cura di G. Scalia. Rocca San Casciano: Cappelli, 1965.

—. Spartaco: tragedia inedita, curata dal manoscritto autografo con una nota di

V. Errante. Lanciano: Carabba, 1919.

—. Teatro, a cura di E. Faccioli. Torino: Einaudi, 1962.

Nievo, Stanislao. Il prato in fondo al mare. Milano: Mondadori, 1975.

Nozzoli, Anna. Immagini di Nievo nel Novecento. Modena: Mucchi, 1995.

Portinari, Folco. Ippolito Nievo. Storia e ideologia. Milano: Silva, 1969.

Ruffilli, Paolo. L'isola e il sogno. Roma: Fazi, 2011.

Samaritani, Fausta. Ippolito Nievo. I giorni sommersi. Venezia: Marsilio, 1996.

Sanguineti, Edoardo. "La macchina narrativa dell'Ariosto". In Il chierico organico.

Scritture e intellettuali. Milano: Feltrinelli, 2000. 65-71.

Sanguinetti Katz, Giuliana. "A psychoanalytic interpretation of the life of Ippolito

Nievo". Italian Quarterly, 22. 84 (1981): 39-50.

—. The Uses of the Myth in Ippolito Nievo. Ravenna: Longo, 1980.

Segatori, Stefania. Forme, temi e motivi della narrativa di Ippolito Nievo. Firenze:

Olschki, 2011.

Solitro, Giuseppe. Ippolito Nievo. Studio biografico con documenti inediti. Padova:

Tipografia del Seminario, 1936.

Titta Rosa, Giovanni. Nievo. Uomo e poeta del Risorgimento. Sarzana: Carpena, 1961.

Ulivi, Ferruccio. Il Romanticismo di Ippolito Nievo. Roma: Anonima Veritas, 1947. Zinna, Lucio. Come un sogno incredibile. Ipotesi sul caso Nievo. Pisa: Giardini, 1980.

—. Il Caso Nievo. Morte di un Garibaldino. Marina di Minturno: Caramanica Editore, 2006. 